PROCEEDINGS OF THE

AMERICAN MATHEMATICAL SOCIETY

Volume 140, Number 9, September 2012, Pages 2937-2944

S 0002-9939(2012)10934-7

Article electronically published on April 11, 2012

\title{
CASTELNUOVO-MUMFORD REGULARITY AND REDUCTION NUMBER OF SMOOTH MONOMIAL CURVES IN $\mathbb{P}^{5}$
}

\author{
MAX JOACHIM NITSCHE \\ (Communicated by Irena Peeva)
}

\begin{abstract}
We will compute explicitly the Castelnuovo-Mumford regularity and the reduction number of coordinate rings of smooth projective monomial curves in $\mathbb{P}^{5}$. Moreover we will show that these numbers are equal.
\end{abstract}

\section{INTRODUCTION}

Let $K$ be a field and $A:=\left\{0, a_{1}, \ldots, a_{d}, \alpha\right\}$ a subset of $\mathbb{N}$, where $d \in \mathbb{N}^{+}$ and $0<a_{1}<\ldots<a_{d}<\alpha$ is a sequence of relatively prime integers. The ring $K[A]:=K\left[t_{0}^{\alpha}, t_{0}^{\alpha-a_{1}} t_{1}^{a_{1}}, \ldots, t_{0}^{\alpha-a_{d}} t_{1}^{a_{d}}, t_{1}^{\alpha}\right] \subseteq K\left[t_{0}, t_{1}\right]$ is uniquely determined by $A$ and isomorphic to the coordinate ring of a projective monomial curve of degree $\alpha$ in $\mathbb{P}^{d+1}$. By a result of Gruson-Lazarsfeld-Peskine [3] the Eisenbud-Goto conjecture [2] holds for projective monomial curves; i.e., the Castelnuovo-Mumford regularity $\operatorname{reg} K[A]$ is bounded by $\alpha-d$. In terms of gaps (see Definition 2.2) $\alpha-d=\sum(\# L)+1$, where $L$ runs over all gaps of $A$. As we see, the bound $\operatorname{reg} K[A] \leq \# L+\# L^{\prime}+1$ given by $L^{\prime}$ vovsky in [6, Proposition 5.5], where $L$ and $L^{\prime}$ are the longest and the second longest gap of $A$, is better than $\alpha-d$ (see [4, Introduction]). It is well known that the projective monomial curve is smooth if and only if $a_{1}=1$ and $a_{d}=\alpha-1$. For smooth projective monomial curves, HellusHoa-Stückrad showed in [4, Theorem 2.7] that $\operatorname{reg} K[A]$ is bounded by $\# L+1$, where $L$ is the longest gap of $A$.

In Section 3 we will compute explicitly the Castelnuovo-Mumford regularity $\operatorname{reg} K[A]$ and the reduction number $\mathrm{r}(K[A]$ ) (see Definition 2.9) of smooth projective monomial curves in $\mathbb{P}^{5}$. For $K[A]=K\left[t_{0}^{\alpha}, t_{0}^{\alpha-1} t_{1}, t_{0}^{\alpha-b} t_{1}^{b}, t_{0}^{\alpha-c} t_{1}^{c}, t_{0} t_{1}^{\alpha-1}, t_{1}^{\alpha}\right]$ defined by $A=\{0,1, b, c, \alpha-1, \alpha\} \subseteq \mathbb{N}$ with $1<b<c<\alpha-1$ we will show (see Corollary 3.9):

$$
\operatorname{reg} K[A]=\mathrm{r}(K[A])=\max \left\{\left\lfloor\frac{c}{b}\right\rfloor+b-2,\left\lfloor\frac{\alpha-b}{\alpha-c}\right\rfloor+\alpha-c-2\right\} .
$$

In view of [5. Theorem 1.1], the property $\operatorname{reg} K[A]=\mathrm{r}(K[A])$ is very interesting, since the Eisenbud-Goto conjecture holds in this situation by [5]. But even for smooth projective monomial curves $\operatorname{reg} K[A]=\mathrm{r}(K[A])$ does not hold in general. In $\mathbb{P}^{d+1}$ for $d=2,3$ we have equality (see [4, Section 3]); of course, we proved the latter property in $\mathbb{P}^{5}$. There are examples of smooth projective monomial curves in $\mathbb{P}^{d+1}$ with $d \geq 6$ and $\mathrm{r}(K[A])<\operatorname{reg} K[A]$; one is mentioned in [4, Example 3.2]. Moreover our interest came from the fact that still the latest bound in 4 for smooth

Received by the editors May 25, 2010.

2010 Mathematics Subject Classification. Primary 13D45.

(C)2012 American Mathematical Society 2937

Reverts to public domain 28 years from publication 
projective monomial curves is imprecise. If we take a look at an example, for the coordinate ring $K[A]$ defined by $A=\{0,1,100,100100,102101,102102\}$ we get by [4] that $\operatorname{reg} K[A] \leq 100000$. On the other hand we know by Corollary 3.9 that $\operatorname{reg} K[A]=2050$. In Section 2 we consider smooth projective monomial curves in $\mathbb{P}^{d+1}$. For unspecified notation, we refer to [1].

\section{Smooth PRojective monomial CURVES in $\mathbb{P}^{d+1}$}

In this section we assume that $K[A]$ is defined by $A:=\left\{0, a_{1}, \ldots, a_{d}, \alpha\right\}$ (see the Introduction) with $a_{1}:=1$ and $a_{d}:=\alpha-1$; i.e., we consider smooth projective monomial curves. Letting $m \in \mathbb{N}^{+}$we set $m A:=A+\ldots+A$ ( $m$ times). Then we have $m A \subseteq\{0,1,2, \ldots, m \alpha-1, m \alpha\}=[0, m \alpha] \cap \mathbb{N}$, and there exists an $m \in \mathbb{N}^{+}$ such that the equality $\#(m A)=m \alpha+1$ holds; in the latter case we say that $m A$ is full. We define $\operatorname{reg}(A):=\min \left\{m \in \mathbb{N}^{+} \mid m A\right.$ is full $\}$. By [4, Corollary 2.5] and Lemma 2.8 we have

$$
\operatorname{reg} K[A]=\operatorname{reg}(A)
$$

As we see, it comes down to a pure combinatorial problem.

Definition 2.1. Let $X$ be a nonempty finite subset of $\mathbb{N}$. We set $\hat{x}:=\min X$ and $\bar{x}:=\max X$. The set $X$ is called an interval if $X=\{\hat{x}, \hat{x}+1, \ldots, \bar{x}-1, \bar{x}\}$, i.e. $X=[\hat{x}, \bar{x}] \cap \mathbb{N}$. We denote such intervals in $\mathbb{N}$ by $[u, v]$ with $u, v \in \mathbb{N}, u \leq v$.

Definition 2.2. A gap $L$ of $X$ is a maximal interval contained in the complement $G_{X}:=[\hat{x}, \bar{x}] \backslash X$ of $\bar{X}$ in $[\hat{x}, \bar{x}]$ and its length is $\# L$. By $\Lambda_{X}$ we denote the set of

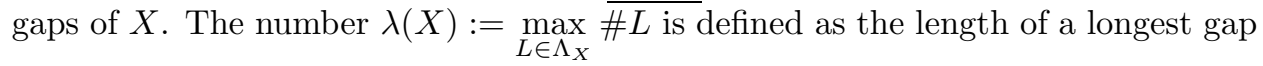
of $X$. We say that $X$ is full if $G_{X}=\emptyset$, i.e., if $X$ is an interval.

For instance $A=\{0,1,5,9,10\}$ has two gaps, i.e. $\{[2,4] \cup[6,8]\}=G_{A}$. Clearly if $m A$ is full for $m \in \mathbb{N}^{+}$, then $(m+i) A$ is also full for every $i \in \mathbb{N}$.

Definition 2.3. For $t \in \mathbb{N}$, let $\kappa_{t}:[0, t] \rightarrow[0, t]$ be the map given by $\kappa_{t}(i):=t-i$, for all $i \in[0, t]$. Note that $\kappa_{t}$ is an order-reversing bijection respecting intervals with $\kappa_{t}^{2}=i d_{[0, t]}$. For $m, i \in \mathbb{N}^{+}$with $i \leq m$, we define $(m A)_{i}:=[(i-1) \alpha, i \alpha] \cap m A$ and $\epsilon:=\max \{r \in \mathbb{N} \mid\{[0, r] \cup[\alpha-r, \alpha]\} \subseteq A\}$.

As we see for smooth projective monomial curves, $\epsilon \geq 1$.

Remark 2.4. With the notation of Definition 2.3, we get that $m A$ is full if and only if $(m A)_{i}$ are full for all $i \in \mathbb{N}$ with $1 \leq i \leq m$ and we have

$$
\begin{gathered}
{[(i-1) \alpha,(i-1) \alpha+\epsilon]=[0, \epsilon]+(i-1) \alpha \subseteq(m A)_{i},} \\
{[i \alpha-\epsilon, i \alpha]=[\alpha-\epsilon, \alpha]+(i-1) \alpha \subseteq(m A)_{i} .}
\end{gathered}
$$

Notation 2.5. We set $\hat{A}:=\kappa_{\alpha}(A)$ and

$$
A_{P}:=\left\{(\alpha, 0),(\alpha-1,1),\left(\alpha-a_{2}, a_{2}\right), \ldots,\left(\alpha-a_{d-1}, a_{d-1}\right),(1, \alpha-1),(0, \alpha)\right\} .
$$

Letting $m \in \mathbb{N}^{+}, i \in \mathbb{N}$ with $i \leq m \alpha$, we define $g_{i}^{m}:=(m \alpha-i, i)$.

Remark 2.6. Let $m \in \mathbb{N}^{+}$. We set $m A_{P}:=A_{P}+\ldots+A_{P}$ ( $m$ times) with the usual addition of tuples. Let $\mu_{m}: m A \rightarrow m A_{P}$ be the map given by $\mu_{m}(i):=g_{i}^{m}$ for all $i \in m A$. Then $\mu_{m}$ is bijective and therefore $\mu_{m}(m A)=m A_{P}$. 
Definition 2.7. We define $G_{m A_{P}}:=\mu_{m}\left(G_{m A}\right)$. A subset of $G_{m A_{P}}$ is called a gap of $m A_{P}$ if it is of the form $\mu_{m}(L)$ with a gap $L$ of $m A$. We say that $m A_{P}$ is full if $G_{m A_{P}}=\emptyset$ and we define $\operatorname{reg}\left(A_{P}\right):=\min \left\{m \in \mathbb{N}^{+} \mid m A_{P}\right.$ is full $\}$.

Lemma 2.8. Let $m, i \in \mathbb{N}^{+}$with $i \leq m$ and $u, v \in \mathbb{N}$ with $u \leq v$. Then

(i) $[u, v] \subseteq m A$ if and only if $[m \alpha-v, m \alpha-u] \subseteq m \kappa_{\alpha}(A)$.

(ii) $(m A)_{i}$ is full if and only if $(m \hat{A})_{m+1-i}$ is full.

(iii) $\operatorname{reg}(A)=\operatorname{reg}(\hat{A})=\operatorname{reg}\left(A_{P}\right)$.

Proof. (i) We have $\kappa_{m \alpha}(m A)=m \kappa_{\alpha}(A)$. Now $(i)$ follows by the fact that $\kappa_{m \alpha}$ is bijective and $\kappa_{m \alpha}([u, v])=[m \alpha-v, m \alpha-u]$.

(ii) Follows from $(i)$ replacing $[u, v]$ by $[(i-1) \alpha, i \alpha]$. (ii).

(iii) By Definition 2.7 we have $\operatorname{reg}(A)=\operatorname{reg}\left(A_{P}\right)$, and now (iii) follows from

Definition 2.9. By [4, Section 3] and the bijective map $\mu_{m}$ the reduction number $\mathrm{r}(K[A])$ of $K[A]$ can be computed by

$$
\mathrm{r}(K[A]):=\min \left\{r \in \mathbb{N}^{+} \mid(r+1) A=r A+\{0, \alpha\}\right\} .
$$

Definition 2.10. We will call $A$ saturated if either $\operatorname{reg}(A)=1$ or $\operatorname{reg}(A)>1$ and

$$
G_{(\operatorname{reg}(A)-1) A} \cap\{[0, \alpha] \cup[(\operatorname{reg}(A)-2) \alpha,(\operatorname{reg}(A)-1) \alpha]\} \neq \emptyset .
$$

Let us take a look at an example: for $K[A]$ defined by $A=$

$$
\{0,1,3,5,11,14,18,21,25,34,44,67,90,94,101,103,108,110,111,113,115,116\},
$$

we have $\mathrm{r}(K[A])=3$ and $\operatorname{reg}(A)=4$. One can check that $\{190\}$ is the last gap in $3 A$, and therefore $A$ is not saturated.

Proposition 2.11. If $A$ is saturated, then $\mathrm{r}(K[A])=\operatorname{reg}(A)$.

Proof. By definition we get that $1 \leq \mathrm{r}(K[A]) \leq \operatorname{reg}(A)$. We therefore may assume that $\operatorname{reg}(A)>1$. Since $A$ is saturated and by Remark 2.4 there exists an $i \notin$ $(\operatorname{reg}(A)-1) A$ with $i \in[2, \alpha-2]$ or with $i \in[(\operatorname{reg}(A)-2) \alpha+2,(\operatorname{reg}(A)-1) \alpha-2]$ $(\epsilon \geq 1)$. By this we have

$$
(\operatorname{reg}(A)-1) A+\{0, \alpha\} \neq[0, \operatorname{reg}(A) \alpha]=(\operatorname{reg}(A)-1+1) A .
$$

Hence $(r+1) A \neq r A+\{0, \alpha\}$ for $r \in \mathbb{N}$ with $1 \leq r \leq \operatorname{reg}(A)-1$, and therefore we get $\mathrm{r}(K[A])>\operatorname{reg}(A)-1$.

Lemma 2.12. Let $i, m \in \mathbb{N}^{+}$with $i \leq m$ and $t \in \mathbb{N}$. If $\lambda\left((m A)_{i}\right) \leq t$, then

$$
\max \left\{\lambda\left(((m+1) A)_{i}\right), \lambda\left(((m+1) A)_{i+1}\right)\right\} \leq \max \{t-\epsilon, 0\} .
$$

Proof. Suppose on the contrary that

$$
\max \left\{\lambda\left(((m+1) A)_{i}\right), \lambda\left(((m+1) A)_{i+1}\right)\right\}>\max \{t-\epsilon, 0\} ;
$$

i.e. there is a gap $L$ of $((m+1) A)_{i}$ or there is a gap $L$ of $((m+1) A)_{i+1}$ such that $\# L>\max \{t-\epsilon, 0\} \geq 0, L=[u, v]$ with $u, v \in \mathbb{N}, u \leq v$. In each of these cases we shall lead to a contradiction.

Case 1. $L \subseteq G_{((m+1) A)_{i+1}}$ : In this case, since $L$ is a gap of $((m+1) A)_{i+1}$ we get by Remark 2.4 that

$$
i \alpha+\epsilon<u \leq v<(i+1) \alpha-\epsilon
$$


Now suppose that $j \in\left\{[u-\alpha, v-\alpha] \cap(m A)_{i}\right\} \neq \emptyset$. Then $j+\alpha \in((m+1) A)_{i+1}$, a contradiction. Hence $[u-\alpha, v-\alpha] \subseteq G_{(m A)_{i}}$.

Now suppose that $\left\{[v-\alpha+1, v-\alpha+\epsilon] \cap(m A)_{i}\right\} \neq \emptyset$. Then one can show that $v \in((m+1) A)_{i+1}($ since $[\alpha-\epsilon, \alpha-1] \subseteq A)$, a contradiction. Hence $[v-\alpha+1, v-$ $\alpha+\epsilon] \subseteq G_{(m A)_{i}}$. This proves that $[u-\alpha, v-\alpha+\epsilon] \subseteq G_{(m A)_{i}}$ which contradicts $\lambda\left((m A)_{i}\right) \leq t$.

Case 2. $L \subseteq G_{((m+1) A)_{i}}$ : By a similar argument, we get $[u-\epsilon, v] \subseteq G_{(m A)_{i}}$.

Lemma 2.13. Let $i, m \in \mathbb{N}^{+}, t \in \mathbb{N}$ with $i \leq m$ and $\lambda\left((m A)_{i}\right) \leq t$. Then the sets $((m+p) A)_{i}, \ldots,((m+p) A)_{i+p}$ are full for $p=\left\lfloor\frac{t-1}{\epsilon}\right\rfloor+1$ and for $p=t$.

Proof. Using Lemma 2.12 by induction on $p \in \mathbb{N}$ one can show that

$$
\max \left\{\lambda\left(((m+p) A)_{i}\right), \ldots, \lambda\left(((m+p) A)_{i+p}\right)\right\} \leq \max \{t-p \epsilon, 0\} .
$$

Hence we get the assertion for $p=t$. The case $p=\left\lfloor\frac{t-1}{\epsilon}\right\rfloor+1$ follows from

$$
t-\left(\left\lfloor\frac{t-1}{\epsilon}\right\rfloor+1\right) \epsilon<t-\left(\frac{t-1}{\epsilon}\right) \epsilon=1 .
$$

Remark 2.14. Lemma 2.13 is a new proof of [4, Theorem 2.7]. Of course this lemma is more general and will be very useful in Section 3 . With $\lambda\left((1 A)_{1}\right)=\lambda(A)$ we get that $((1+q) A)_{1}, \ldots,((1+q) A)_{1+q}$ are full, and therefore $(1+q) A$ is full for $q=\left\lfloor\frac{\lambda(A)-1}{\epsilon}\right\rfloor+1$. Hence

$$
\operatorname{reg} K[A] \stackrel{(1)}{=} \operatorname{reg}(A) \leq\left\lfloor\frac{\lambda(A)-1}{\epsilon}\right\rfloor+2 .
$$

As a direct result, $\operatorname{reg} K[A]$ is also bounded by $\lambda(A)+1$.

It is known that the bound of [4, Theorem 2.7] is sharp for smooth projective monomial curves in $\mathbb{P}^{d+1}$ for $d=2,3$ (see [4, Section 3]). Now we will establish two basic combinatorial lemmas which will be useful to prove Theorem 3.1 .

Notation 2.15. From now on we set $m:=\left\lfloor\frac{a_{3}}{a_{2}}\right\rfloor, x:=m+a_{2}-2$ and we assume that $d \geq 4$ (see Introduction).

Lemma 2.16. If $(x-1)>0$, then $\left(a_{2} m-1\right) \notin(x-1) A$.

Proof. We have $a_{2} m-1 \leq a_{2} \frac{a_{3}}{a_{2}}-1=a_{3}-1$. Suppose that $a_{2} m-1 \in(x-1) A$. Then there are $\beta_{1}, \beta_{2} \in \mathbb{N}$ such that

$$
a_{2} m-1=\beta_{1}+\beta_{2} a_{2}
$$

and

$$
\beta_{1}+\beta_{2} \leq x-1=m+a_{2}-3,
$$

and we get $\left(a_{2}-1\right)\left(m-\beta_{2}-1\right)=a_{2} m-a_{2} \beta_{2}-a_{2}-m+\beta_{2}+1 \stackrel{(3)}{=} \beta_{1}+\beta_{2}-m-a_{2}+2$

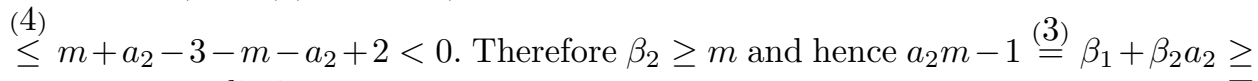
$a_{2} m$, a contradiction.

Lemma 2.17. If $\lambda(A)=a_{3}-a_{2}-1$, then $(x A)_{1}, \ldots,(x A)_{a_{2}-1}$ are full. 
Proof. We will now show that $\lambda\left((m A)_{1}\right) \leq a_{2}-2$ and we are done by Lemma 2.13, Let $i \in \mathbb{N}, 1 \leq i \leq d-1$. We have $\lambda(A)=a_{3}-a_{2}-1$ and therefore

$$
a_{i+1}-a_{i} \leq a_{3}-a_{2} .
$$

Fixing one $i$, we will show that there cannot be a bigger gap than $a_{2}-2$ between $a_{i}$ and $a_{i+1}$ in $(m A)_{1}$ and therefore $\lambda\left((m A)_{1}\right) \leq a_{2}-2$. Let $j \in \mathbb{N}, 1 \leq j \leq m$. Then

$$
\left[a_{i}+(m-j) a_{2}, a_{i}+(m-j) a_{2}+j-1\right] \subseteq m A .
$$

One can check that the distances between these intervals are smaller than or equal to $a_{2}-1$; i.e., the length of every gap until $a_{i}+(m-1) a_{2}$ for $j=1$ is smaller than or equal to $a_{2}-2$. If $a_{i+1} \leq a_{i}+(m-1) a_{2}$, we are done. Let us assume $a_{i}+(m-1) a_{2}<a_{i+1}$. Then we have to show that the distance between these numbers is smaller than or equal to $a_{2}-1$, but this is clear by (6) :

$$
a_{2}-1-a_{i+1}+\underbrace{a_{i}}_{\geq a_{i+1}-a_{3}+a_{2}}+(m-1) a_{2} \underbrace{\sqrt[5]{\geq}(m+1)}_{>\frac{a_{3}}{a_{2}}-1+1} a_{2}-a_{3}-1>-1 .
$$

\section{Smooth projective monomial CuRVES in $\mathbb{P}^{5}$}

In this section we assume that $A:=\{0,1, b, c, \alpha-1, \alpha\}$ with $A \subseteq \mathbb{N}$ and $1<$ $b<c<\alpha-1$. We have $K[A]=K\left[t_{0}^{\alpha}, t_{0}^{\alpha-1} t_{1}, t_{0}^{\alpha-b} t_{1}^{b}, t_{0}^{\alpha-c} t_{1}^{c}, t_{0} t_{1}^{\alpha-1}, t_{1}^{\alpha}\right], \hat{A}=$ $\{0,1, \alpha-c, \alpha-b, \alpha-1, \alpha\}, m=\left\lfloor\frac{c}{b}\right\rfloor$ and $x=m+b-2$. Our aim is to show:

\section{Theorem 3.1.}

$$
\operatorname{reg}(A)=\max \left\{\left\lfloor\frac{c}{b}\right\rfloor+b-2,\left\lfloor\frac{\alpha-b}{\alpha-c}\right\rfloor+\alpha-c-2\right\} .
$$

Remark 3.2. We note that the symmetric case, i.e. $c=\alpha-b$, was proven in 4 , Proposition 3.5]. Let us assume that $A$ is full. Then $A=\{0,1,2,3,4,5\}$ and we get that $\operatorname{reg}(A)=1=\max \{1,1\}$, and hence (7) holds. Without loss of generality we therefore may assume that $A$ has at least one gap, i.e. $\lambda(A)>0$. By this we have $b>2$ if $\lambda(A)=b-2$ and $x-1>0$ if $x \geq y$.

Notation 3.3. For the next lemmas we set $n:=\left\lfloor\frac{\alpha-b}{\alpha-c}\right\rfloor, y:=n+\alpha-c-2$ and $i:=x-y$.

Lemma 3.4. (i) If $\lambda(A)=b-2$, then (7) holds.

(ii) If $\lambda(A)=\alpha-c-2$, then (7) holds.

Proof. We will only prove $(i)$. Then $(i i)$ follows by replacing $A$ by $\hat{A}$. It is clear that $\lambda\left((1 A)_{1}\right)=\lambda(A)=b-2$ and we get that $(b-1) A$ is full, by Lemma 2.13 , Since $\lambda(A)=b-2$ we have $b-1 \geq c-b$, i.e. $2 b-1 \geq c$. Then

$$
m=\left\lfloor\frac{c}{b}\right\rfloor=1,
$$

since $c>b$. Hence $x=m+b-2 \stackrel{8}{=} b-1$ and $(b-2) A$ is not full by Lemma 2.16 . Therefore

$$
\operatorname{reg}(A)=b-1=x .
$$

Suppose that $x<y$. Then by Lemma 2.16

$$
(\alpha-c) n-1 \notin(y-1) \hat{A}
$$

hence $\operatorname{reg}(A) \stackrel{2.8}{=} \operatorname{reg}(\hat{A}) \stackrel{96}{>} y-1 \geq x$, a contradiction. This proves $x \geq y$. 
So we only have to consider the case $\lambda(A)=c-b-1$, or in other words $c-b \geq b-1$ and $c-b \geq \alpha-1-c$. In the following we will show that (7) holds for $x \geq y$, and this will finish the proof of Theorem 3.1 , since otherwise one can replace $A$ by $\hat{A}$.

Lemma 3.5. If $\lambda(A)=c-b-1$ and $x \geq y$, then $(x A)_{1}, \ldots,(x A)_{b-1}$ and $(x A)_{n}, \ldots,(x A)_{x}$ are full.

Proof. By Lemma 2.17 we have the first assertion and by symmetry we get that

$$
(y \hat{A})_{1}, \ldots,(y \hat{A})_{\alpha-c-1}
$$

are full. Let $h \in \mathbb{N}, 0 \leq h \leq i=x-y$ and $j \in(y \hat{A})_{\alpha-c-1}$. Then $j+h \alpha \in x \hat{A}$; hence $(x \hat{A})_{1}, \ldots,(x \hat{A})_{\alpha-c-1+i}$ are full. Note that

$$
x=y+i=n+\alpha-c-2+i \Longleftrightarrow x+1-n=\alpha-c-1+i .
$$

By (10) this is equivalent to $(x \hat{A})_{x+1-x}, \ldots,(x \hat{A})_{x+1-n}$ being full, and therefore

$$
(x A)_{n}, \ldots,(x A)_{x}
$$

are full by Lemma 2.8 .

We will now show that $(x A)_{\psi}$ is full for $b \leq \psi \leq n-1$, since our goal is to prove that $x A$ is full. Without loss of generality we therefore may assume that $b \leq n-1$. First of all we will give a construction of two intervals contained in $x A$; after this we will show that there cannot be a natural number between these intervals and therefore $(x A)_{\psi}$ have to be full.

Lemma 3.6. If $\psi \in \mathbb{N}, b \leq \psi \leq n-1$ and $x \geq y$, then

$$
\{[(\psi-1) \alpha,(\psi-1) \alpha+(x-\psi+1) b] \cup[\psi c, \psi \alpha]\} \subseteq x A .
$$

Proof. Letting $k \in \mathbb{N}$ with $0 \leq k \leq(x-\psi+1)$, we get

$$
(\psi-1) \alpha+(x-\psi+1-k) b \in x A .
$$

We will take an element $b^{*}=(x-\psi+1-k) b$ for one fixed $k$. Let $h \in \mathbb{N}$, with $0 \leq h \leq b-1$. Then

$$
\underbrace{(\psi-1-h)}_{\geq 0} \alpha+h(\alpha-1)+b^{*} \in x A
$$

since $\psi \geq b$. Therefore

$$
[(\psi-1) \alpha,(\psi-1) \alpha+(x-\psi+1) b] \subseteq x A .
$$

Note that it is not necessary at this point to bound $\psi$ by $\psi \leq n-1$. The above argument also holds for $\psi \leq x$. By the same argument we have

$$
[(\phi-1) \alpha,(\phi-1) \alpha+(x-\phi+1)(\alpha-c)] \subseteq x \hat{A}
$$

with $\phi \in \mathbb{N}, \alpha-c \leq \phi \leq x$. By Lemma 2.8 and $\kappa_{x \alpha}^{2}=i d_{[0, x \alpha]}$ we get (11) $[(x-\phi+1) c,(x-\phi+1) \alpha]=\kappa_{x \alpha}([(\phi-1) \alpha,(\phi-1) \alpha+(x-\phi+1)(\alpha-c)]) \subseteq x A$.

We know that $\alpha-c \leq \phi \leq x$ and $\psi$ is bounded by $b \leq \psi \leq n-1$. Let $\phi=\alpha-c$. Hence $x-\phi+1=n+\alpha-c-2+i-\alpha+c+1=n-1+i$, with $i=x-y \geq 0$. Let $\phi=x$. Hence $x-\phi+1=1$. Therefore for all $\psi$ there exists a $\phi$ with $x-\phi+1=\psi$ (since $b \geq 2$ ). Then by (11),

$$
[\psi c, \psi \alpha] \subseteq x A .
$$

Lemma 3.7. If $\psi \in \mathbb{N}, b \leq \psi \leq n-1$ and $x \geq y$, then $(x A)_{\psi}$ is full. 
Proof. We will show that

$$
\psi c-((\psi-1) \alpha+(x-\psi+1) b)=: z=-x b-\psi(\alpha-c-b)+\alpha-b<2,
$$

i.e. $z \leq 1$, since $z \in \mathbb{Z}$. This implies that

$$
[(\psi-1) \alpha, \psi \alpha] \stackrel{\sqrt[12]{\subseteq}}{\subseteq}\{[(\psi-1) \alpha,(\psi-1) \alpha+(x-\psi+1) b] \cup[\psi c, \psi \alpha]\} \stackrel{3.6}{\subseteq} x A,
$$

and hence $(x A)_{\psi}=[(\psi-1) \alpha, \psi \alpha] \cap x A=[(\psi-1) \alpha, \psi \alpha]$; i.e. $(x A)_{\psi}$ is full. To prove (12) we collect the following inequalities:

$$
\begin{gathered}
b \leq \psi \leq n-1, \\
n>\frac{\alpha-b}{\alpha-c}-1, \quad m>\frac{c}{b}-1, \\
m+b-2=x \geq y=n+\alpha-c-2, \\
b \geq 2, \quad \alpha-c \geq 2 .
\end{gathered}
$$

Case 1. $(\alpha-c-b)>0$ :

$$
\begin{array}{ll}
\stackrel{13}{\leq} & -x b-b(\alpha-c-b)+\alpha-b \\
= & -b(m+b-2+\alpha-c-b+1)+\alpha \\
\stackrel{14}{<} & -b\left(\frac{c}{b}+\alpha-c-2\right)+\alpha \\
= & -b(\alpha-c)+\alpha-c+2 b \\
= & (1-b)(\alpha-c)+2 b \\
\stackrel{16}{\leq} & 2-2 b+2 b=2 .
\end{array}
$$

Case 2. $(\alpha-c-b) \leq 0$ :

$$
\begin{array}{ll}
\underset{\frac{15}{\leq}}{\frac{15}{\leq}} & -b(n+\alpha-c-2)+\psi(b+c-\alpha)+\alpha-b \\
\stackrel{13}{\leq} & -b(n-1)-b(\alpha-c-1)+(n-1)(b+c-\alpha)+\alpha-b \\
= & (n-1)(c-\alpha)-b(\alpha-c-1)+\alpha-b \\
\stackrel{14}{<} & \left(\frac{\alpha-b}{\alpha-c}-2\right)(c-\alpha)+b(c-\alpha)+b+\alpha-b \\
= & (c-\alpha)(b-2)+b \\
\underline{16} & -2 b+4+b \\
\stackrel{116}{\leq} & 2 .
\end{array}
$$

Proof of Theorem 3.1 .

If $\lambda(A)=b-2$ or $\lambda(A)=\alpha-c-2$, the assertion follows from Lemma 3.4.

If $\lambda(A)=c-b-1$ and $x \geq y$, then by Lemmas 3.5 and 3.7. $(x A)_{1}, \ldots,(x A)_{x}$ are full. Therefore we get that $x A$ is full. By Lemma 2.16, $(x-1) A$ is not full and hence

$$
\operatorname{reg}(A)=x
$$

Similarly for $y \geq x$ and $\lambda(A)=\lambda(\hat{A})=c-b-1$, replace $A$ by $\hat{A}$.

Corollary 3.8. A is saturated. 
Proof. Without loss of generality assume that $\operatorname{reg}(A)>1$. If $\operatorname{reg}(A)=x$, then $A$ is saturated by Lemma 2.16. If $\operatorname{reg}(A)=y$, then $\hat{A}$ is saturated by Lemma 2.16. Hence $A$ is saturated by Lemma 2.8 and we are done.

\section{Corollary 3.9.}

$$
\begin{aligned}
& \operatorname{reg} K[A]=\mathrm{r}(K[A])=\max \left\{\left\lfloor\frac{c}{b}\right\rfloor+b-2,\left\lfloor\frac{\alpha-b}{\alpha-c}\right\rfloor+\alpha-c-2\right\} . \\
& \text { Proof. } \max \left\{\left\lfloor\frac{c}{b}\right\rfloor+b-2,\left\lfloor\frac{\alpha-b}{\alpha-c}\right\rfloor+\alpha-c-2\right\} \stackrel{3.1}{=} \operatorname{reg}(A) \frac{3.8}{\underline{\underline{\underline{1}}}} \mathrm{r}(K[A]) \\
& =\operatorname{reg}(A) \stackrel{11}{=} \operatorname{reg} K[A] \text {. }
\end{aligned}
$$

\section{ACKNOWLEDGEMENTS}

The author would like to thank Jürgen Stückrad, Dilip Patil and Michael Hellus for many helpful discussions.

\section{REFERENCES}

1. Eisenbud, D., The Geometry of Syzygies, Springer, Berlin, 2005. MR2103875 (2005h:13021)

2. Eisenbud, D. and Goto, S., Linear free resolutions and minimal multiplicity, J. Algebra $\mathbf{8 8}$ (1984), no. 1, 89-133. MR741934 (85f:13023)

3. Gruson, L., Lazarsfeld, R. and Peskine, C., On a theorem of Castelnuovo and the equations defining space curves, Invent. Math. 72 (1983), no. 3, 491-506. MR704401 (85g:14033)

4. Hellus, M., Hoa, L. T. and Stückrad, J., Castelnuovo-Mumford regularity and the reduction number of some monomial curves, Proc. Amer. Math. Soc. 138 (2010), no. 1, 27-35. MR2550167 (2010j:13035)

5. Hoa, L. T. and Stückrad, J., Castelnuovo-Mumford regularity of simplicial toric rings, J. Algebra 259 (2003), no. 1, 127-146. MR 1953712(2003j:13024)

6. L'vovsky, S., On inflection points, monomial curves and hypersurfaces containing projective curves, Math. Ann. 306 (1996), no. 4, 719-735. MR.1418349 (99e:14033)

Max-Planck-Institute for Mathematics in the Sciences, Inselstrasse 22, 04103 LeipZIG, Germany

E-mail address: nitsche@mis.mpg.de 